\title{
On balanced separators, treewidth, and cycle rank
}

\author{
Hermann Gruber
}

\begin{abstract}
We investigate relations between different width parameters of graphs, in particular balanced separator number, treewidth, and cycle rank. Our main result states that a graph with balanced separator number $k$ has treewidth at least $k$ but cycle rank at most $k \cdot\left(1+\log \frac{n}{k}\right)$, thus refining the previously known bounds, as stated by Robertson and Seymour (1986) and by Bodlaender et al. (1995). Furthermore, we show that the improved bounds are best possible.
\end{abstract}

AMS 2010 SUBJECT CLASSIFICATIONS: 05C40, 05 C35.

KEYWORDS AND PHRASES: Vertex separator, treewidth, pathwidth, bandwidth, cycle rank, ordered coloring, vertex ranking, hypercube.

\section{Preliminaries}

Throughout this paper, $\log x$ denotes the binary logarithm of $x$, and $\ln x$ denotes the natural logarithm of $x$.

\subsection{Vertex separators}

We assume the reader is familiar with basic notions in graph theory, as contained in [8]. In a connected graph $G$, a subset $X$ of the vertex set $V(G)$ is a vertex separator for $G$, if there exists a pair $x, y$ of vertices lying in distinct components of $V(G)-X$, or if $V(G)-X$ contains less than two vertices. Beside vertex separators, also so-called edge separators are studied in the literature. As we shall deal only with the former kind of separators, we will mostly speak just of separators when referring to vertex separators.

If $G$ has several connected components, we say $X$ is a separator for $G$ if it is a separator for some component of $G$. A separator $X$ is (inclusion) minimal if no other separator is properly contained in it. A separator $X$ is balanced, if every component of $G \backslash X$ contains at most $\left\lceil\frac{|V(G)|-|X|}{2}\right\rceil$ vertices; and it is called strictly balanced, if every component of $G \backslash X$ contains at most $\frac{|V(G)|-|X|}{2}$ vertices.

arXiv: 1012.1344 
Lemma 1.1. Let $G$ be a graph with a balanced separator of size at most $k$. Then $G$ has a balanced separator of size exactly $k$.

Proof. Assume $X$ is a balanced separator of size $x<|V(G)|$. Then we can extend $S$ to a balanced separator of size $x+1$ as follows: Let $C_{1}, C_{2}, \ldots$ be the components of $G-X$, ordered by decreasing cardinality. Let $v$ be any vertex in the largest component $C_{1}$. We claim that $X \cup\{v\}$ is again a balanced separator for $G$. Namely, we have

$$
\left|C_{1} \backslash\{v\}\right| \leq\left\lceil\frac{|V(G)|-|X|}{2}\right\rceil-1 \leq\left\lceil\frac{|V(G)|-|X \cup\{v\}|}{2}\right\rceil .
$$

The components $C_{i}$ in $G-X$ with $i \geq 2$ are also components of $G-(X \cup\{v\})$, so it remains to bound the cardinality of $C_{2}$. The latter is the second largest component of $G-X$, thus $\left|C_{2}\right| \leq\left\lfloor\frac{|V(G)|-|X|}{2}\right\rfloor$, and consequently

$$
\left|C_{2}\right| \leq\left\lfloor\frac{|V(G)|-|X \cup\{v\}|+1}{2}\right\rfloor \leq\left\lceil\frac{|V(G)|-|X \cup\{v\}|}{2}\right\rceil .
$$

For the components $C_{i}$ with $i>2$, we have of course $\left|C_{i}\right| \leq\left|C_{2}\right|$, and the proof is completed.

Arguably, the very first result on graph separators, proved by Jordan [14] in the 19th century, is most naturally phrased in terms of non-strictly balanced separators: every tree admits a balanced separator consisting of a single vertex. However, the usage of strictly balanced separators seems predominant in the more recent literature (see e.g. [3, 16, 21]), perhaps for the reason that the use of the ceiling operator $\lceil x\rceil$ seems unappealing. But the monotonicity property stated in Lemma 1.1 ceases to be true for strictly balanced separators. This is already witnessed by very simple examples, such as $P_{2 k+1}$, the path graph of odd order $2 k+1$. In any case, we can always turn a balanced separator into a strictly balanced separator by adding at most one vertex.

The (strict) balanced separator number of a graph $G$, denoted by s $(G)$ (resp. $\tilde{\mathrm{s}}(G)$ for the strict version), is defined as the smallest integer $k$ such that for every $Q \subseteq V(G)$, the induced subgraph $G[Q]$ admits a (strictly) balanced separator of size at most $k$. Observe that for any graph $G$, we have $\mathrm{s}(G) \leq \tilde{\mathrm{s}}(G) \leq \mathrm{s}(G)+1$.

\subsection{Width parameters}

The cycle rank of a graph $G$, denoted by $\mathrm{r}(G)$, is a structural complexity measure on graphs and is inductively defined as follows: If $G$ has no edges, 
then $\mathrm{r}(G)=1$; if $G$ has several components, then $\mathrm{r}(G)$ is the maximum cycle rank among the connected components of $G$; otherwise, $\operatorname{r}(G)=1+$ $\min _{v \in V(G)} \mathrm{r}(G-v)$. It easily follows from the definition of cycle rank that for every vertex $v \in V(G)$ holds $\mathrm{r}(G-v) \leq 1+\mathrm{r}(G)$, and similarly $\mathrm{r}(G-X) \leq$ $|X|+\mathrm{r}(G-X)$ for every vertex subset $X \subseteq V(G)$.

The notion of cycle rank was originally devised by Eggan and Büchi [9] as a notion on digraphs, and appears in the literature under many different names, such as ordered chromatic number [15], vertex ranking number [2], tree-depth [19], or minimum elimination tree height [3, 17]. Although all these notions ultimately refer to the same concept, some sources use a different normalization. For instance, the minimum elimination tree height of a graph is equal to the cycle rank minus 1.

Other structural complexity measures studied in this paper include the treewidth and pathwidth of graphs; more background information on these two measures can be found in [1, 16, 22]. For a graph $G$, let $\mathcal{V}=\left\{U_{1}, U_{2}, \ldots, U_{r}\right\}$ be a collection of subsets of $V(G)$. A tree $\mathcal{T}=(\mathcal{V}, \mathcal{E})$ with vertex set $\mathcal{V}$ is called a tree decomposition, if all of the following hold: (i) The collection $\mathcal{V}$ covers the vertex set of the graph $G$, in the sense that $V=\bigcup_{U \in \mathcal{V}} U$; (ii) For every edge $(u, v) \in E$, there is a tree node $U \in \mathcal{V}$ such that both $u$ and $v$ are in $U$; and (iii) If two tree nodes $U_{1}$ and $U_{2}$ are connected in the tree by a path, then $U_{1} \cap U_{2}$ is a subset of each tree node visited along this path. The width of a tree decomposition $\mathcal{T}=(\mathcal{V}, \mathcal{E})$ is defined as $\max \{|U|-1 \mid U \in \mathcal{V}\}$, and the treewidth of $G$ is defined as the minimum width among all tree decompositions for $G$. A path decomposition is a tree decomposition where $\mathcal{T}$ is required to be a path graph; and the pathwidth of $G$ is the minimum width among all path decompositions for $G$.

\section{Main results}

We recall the following well-known result [3, Thm. 11], which relates the strict balanced separator number $\tilde{\mathrm{s}}(G)$, treewidth $\operatorname{tw}(G)$, pathwidth $\operatorname{pw}(G)$, and the cycle rank $\mathrm{r}(G)$ of a graph $G$ :

Theorem 2.1. Let $G$ be a graph of order $n \geq 2$, let $\tilde{\mathrm{s}}(G)$ denote its strict balanced separator number, let $\operatorname{tw}(G)$ and $\mathrm{pw}(G)$ denote its treewidth and pathwidth, respectively, and let $\mathrm{r}(G)$ denote its cycle rank. Then

$$
\tilde{\mathrm{s}}(G)-1 \leq \mathrm{tw}(G) \leq \operatorname{pw}(G) \leq \mathrm{r}(G) \leq 1+\tilde{\mathrm{s}}(G) \cdot \log n .
$$

As Bodlaender et al. [3] note, the bounds stated in this theorem are spread across the literature, and (variations of) some of these bounds were discovered independently by several groups of authors. 
Known examples of graphs having a logarithmic gap between treewidth and pathwidth are the complete binary trees of order $2^{d}-1$, which have treewidth 1 but pathwidth $\lceil d / 2\rceil$, see $[22,23]$. For the relation between pathwidth and cycle rank, a similar role is played by the path graphs of order $n$, which have pathwidth 1 but cycle rank $1+\lfloor\log n\rfloor$, see [18]. Indeed, the cycle rank of trees of order $n$ can be no larger than this [15]. But observe that the strict balanced separator number of a path of order $n$ equals 2 for $n \geq 4$, so Theorem 2.1 gives only an upper bound of $2 \cdot(1+\log n)$ for all trees of diameter at least 3. Comparing this with the upper bound on the cycle rank of trees [15] noted above, we see that the former bound is a forteriori unsharp by a factor 2 . The situation is even worse for graphs whose balanced separator number is linear in $n$, such as complete graphs or expanders: their cycle rank can trivially be at most $n$. So the estimate is off by a factor of $\Omega(\log n)$ in this case. Therefore, our first aim will be to refine the rightmost inequality of Theorem 2.1.

The following recurrence will play a crucial role in our investigation.

Definition 2.2. For integers $k, n \geq 1$, let $R_{k}(n)$ be given by the recurrence

$$
R_{k}(n)=k+R_{k}\left(\left\lceil\frac{n-k}{2}\right\rceil\right),
$$

with $R_{k}\left(r_{0}\right)=r_{0}$ for $r_{0} \leq k$.

As it turns out, the function $R_{k}(n)$ can serve as upper bound on the cycle rank of a graph in terms of its order and its balanced separator number:

Lemma 2.3. Let $G$ be a graph of order $n$ whose balanced separator number is at most $k$. Then for the cycle rank $\mathrm{r}(G)$ holds

$$
\mathrm{r}(G) \leq R_{k}(n)
$$

Proof. The overall structure of the argument is the same as e.g. in $[3,19]$, but here we derive a somewhat stronger statement. We prove the statement by induction on the order $n$ of $G$. The base cases $n \leq k$ of the induction are easily seen to hold, since the cycle rank of a graph is always bounded above by its order.

For the induction step, assume $n>k$. Let $X$ be a balanced separator for $G$ of size exactly $k$. Using Lemma 1.1, we know that such a separator exists. Denote the connected components of $G-X$ by $C_{1}, \ldots, C_{p}$. Then $\mathrm{r}(G) \leq$ $k+\mathrm{r}(G-X)$, and by definition of cycle rank, $\mathrm{r}(G-X) \leq \max _{i=1}^{p} \mathrm{r}\left(G\left[C_{i}\right]\right)$. As $X$ is a balanced separator, we have $\left|C_{i}\right| \leq\left\lceil\frac{n-k}{2}\right\rceil$ for $1 \leq i \leq p$, so we 
can apply the induction hypothesis to obtain $\max _{i=1}^{p} \mathrm{r}\left(G\left[C_{i}\right]\right) \leq R_{k}\left(\left\lceil\frac{n-k}{2}\right\rceil\right)$. Putting these pieces together, we have $\mathrm{r}(G) \leq k+R_{k}\left(\left\lceil\frac{n-k}{2}\right\rceil\right)$, as desired.

In fact, we shall see in a moment that this bound is best possible for all $k$ and each $n \geq k+1$. As in [6], let $P_{n}^{k}$ denote the the $k$ th power of a path graph of order $n$, in which two distinct vertices $u, v$ in $V\left(P_{n}\right)=1,2, \ldots, n$ are adjacent iff $|v-u| \leq k$. Determining the cycle rank of this graph is a question recently posed by Novotny et al. [20] and subsequently answered by Chang et al. [5]:

Theorem 2.4 (Chang et al.). Let $k, n \in \mathbb{N}$, and let $P_{n}^{k}$ denote the $k$ th power of a path of order $n$. Then

$$
\mathrm{r}\left(P_{n}^{k}\right)=R_{k}(n)
$$

Since the function $R_{k}(n)$ from Definition 2.2 is arguably very important to our context, we will devote more effort to understanding this recurrence. Chang et al. [5] also derive an explicit formula, namely

$$
R_{k}(n)= \begin{cases}n, & \text { if } k \geq n-1 \\ k \cdot\left(\left\lfloor\log \left(1+\frac{n}{k}\right)\right\rfloor-1\right)+\left\lceil\frac{n+k}{\left.2^{\left\lfloor\log \left(1+\frac{n}{k}\right)\right\rfloor}\right\rceil,},\right. & \text { if } k \leq n-2\end{cases}
$$

Now we can compute each value of $R_{k}(n)$ for given values of $k$ and $n$ effortlessly. However, the formula is somewhat unwieldy. This calls for a more convenient expression for reasoning about $R_{k}(n)$. Therefore, we shall derive an easier upper bound in closed form. For each fixed $k$, that upper bound is sharp infinitely often, so our estimate is essentially the best possible.

Theorem 2.5. Let $k, n \geq 1$ be integers. Then

$$
R_{k}(n) \leq k \cdot\left(1+\log \frac{n}{k}\right)
$$

with equality iff $n=k \cdot\left(2^{j}-1\right)$ for some $j \in \mathbb{N} \backslash\{0\}$.

Proof. Instead of analyzing the recurrence $R_{k}(\cdot)$ directly, we first look at the problem from a different perspective. To this end, for a given positive integer $r$, let $N_{k}(r)$ denote the smallest integer $n$ such that $R_{k}(n) \geq r$. Then by $R_{k}(2 r+k-1)=R_{k}(2 r+k)=k+R_{k}(r)$, we have $N_{k}(r+k)=$ $2 \cdot N_{k}(r)+k-1$, and the recurrence terminates with $N_{k}\left(r_{0}\right)=r_{0}$ for $r_{0} \leq k$.

To get a closed form for $N_{k}(r)$, we make some use of finite calculus (see [11]): The backward difference of $N_{k}$, denoted by $\nabla N_{k}$, is defined as 
$\nabla N_{k}(i)=N_{k}(i)-N_{k}(i-1)$. For convenience, let us define $N_{k}(0)=0$, such that $\nabla N_{k}(1)$ is well-defined.

Claim 2.5.1. For all integers $i, j$ with $i \geq 1$ and $(i-1) \cdot k<j \leq i \cdot k$ holds

$$
\nabla N_{k}(j)=2^{i-1} .
$$

Proof. We prove this by induction on $i$. The base cases where $i=1$ and $j \leq k$ are easily verified, so it remains to perform the induction step. For $(i-1) \cdot k<j \leq i \cdot k$, we have

$$
\begin{aligned}
\nabla N_{k}(j) & =2 \cdot N_{k}(j-k)+k-1-2 \cdot N_{k}(j-1-k)-k+1 \\
& =2 \cdot \nabla N_{k}(j-k) \\
& =2 \cdot 2^{i-2}
\end{aligned}
$$

where the last step above holds by induction hypothesis, since $(i-2) \cdot k<$ $j-k \leq(i-1) \cdot k$. This completes the proof of the claim.

Now that we have a closed form for the backward differences, it is not difficult to derive a closed form for $N_{k}(r)$.

Claim 2.5.2. For all integers $k, r \geq 1$ holds

$$
N_{k}(r)=(k+r \bmod k) \cdot 2^{(r-r \bmod k) / k}-k .
$$

Proof. Telescoping sums yields $N_{k}(r)=N_{k}(r)-N_{k}(0)=\sum_{i=1}^{r} \nabla N_{k}(i)$. Write $r$ as $r=k \cdot s+t$ with $s=r \operatorname{div} k$ and $t=r \bmod k$. We arrive at a simplified form for $N_{k}(r)$ by grouping the summands appropriately and by rewriting those using Claim 2.5.1 afterwards:

$$
\begin{aligned}
N_{k}(r) & =\sum_{i=1}^{s} \sum_{\substack{j>(i-1) \cdot k \\
j \leq i \cdot k}} \nabla N_{k}(j)+\sum_{j=s \cdot k+1}^{r} \nabla N_{k}(j) \\
& =\sum_{i=1}^{s}\left(k \cdot 2^{i-1}\right)+t \cdot 2^{s}=k \cdot\left(2^{s}-1\right)+t \cdot 2^{s} . \\
& =(k+r \bmod k) \cdot 2^{(r-r \bmod k) / k}-k .
\end{aligned}
$$

In the last line, we made use of the facts $t=r \bmod k$ and $s=\frac{r-r \bmod k}{k}$. 
Resolving this formula after $r$ would result in the unhandy formula (1) by Chang et al. [5]. We thus resort to giving an upper bound that is tight infinitely often.

Claim 2.5.3. For all integers $k, r$ with $k \geq 2$ and $r \geq 1$ holds

$$
N_{k}(r) \geq k \cdot\left(2^{r / k}-1\right)
$$

with equality iff $r \bmod k=0$.

Proof. To prove our claim, we consider the univariate real-valued function $f_{k, r}$ given by

$$
f_{k, r}(x)=(k+x) \cdot 2^{(r-x) / k}-k,
$$

with $k$ and $r$ positive real numbers greater than 1 . Differentiating after $x$ gives

$$
f_{k, r}^{\prime}(x)=2^{(r-x) / k} \cdot\left(1-\left(1+\frac{x}{k}\right) \cdot \ln 2\right) .
$$

An easy computation yields $f_{k, r}^{\prime}(x) \geq 0$ if and only if $x \leq k \cdot(1 / \ln 2-1)$, so the function $f_{k, r}(x)$ has a unique maximum at $x_{0}=k \cdot(1 / \ln 2-1)$, and it is monotonically increasing (resp. decreasing) for $x<x_{0}$ (resp. $x>x_{0}$ ). Now let us restrict the domain of $f_{k, r}$ to the closed interval $I=[0 ; k-1]$. Since $0<1 / \ln 2-1<1 / 2$, the number $x_{0}$ is contained within $I$ for $k \geq 2$.

Elementary calculus shows that this restricted function will attain its absolute minimum at the left or right boundary of $I$. Let us calculate which one is the case here:

$$
f_{k, r}(k-1)-f_{k, r}(0)=k \cdot 2^{r / k} \cdot\left(\left(2-\frac{1}{k}\right) \cdot 2^{(-k+1) / k}-1\right)
$$

By evaluating the right-hand side at $k=1$ and by differentiating after $k$, we can deduce that this expression is strictly greater than 0 for all realvalued $k>1$. Thus, $f_{k, r}(k-1)>f_{k, r}(0)$, and we conclude that the function $f_{k, r}: I \rightarrow \mathbb{R}$ attains its absolute minimum at $x_{1}=0$.

We return to the function $N_{k}(r)$, whose domain are the positive integers. Recall that Claim (2.5.2) states that

$$
N_{k}(r)=(k+r \bmod k) \cdot 2^{(r-r \bmod k) / k}-k .
$$


Combining this with (8), we get

$$
N_{k}(r)=(k+r \bmod k) \cdot 2^{(r-r \bmod k) / k}-k=f_{k, r}(r \bmod k) .
$$

We saw above that the function $f_{k, r}(\cdot)$, when restricted to the interval $[0 ; k-$ 1], attains its absolute minimum for $x_{1}=0$. So we can deduce that

$$
N_{k}(r) \geq f_{k, r}(0)=k \cdot\left(2^{r / k}-1\right)
$$

holds for all $r \geq 1$ and $k \geq 2$, and that equality holds if and only if $r \bmod k=$ 0 . This completes the proof of the claim.

We are finally in position to analyze the recurrence $R_{k}(n)$. The special case $k=1$ is well known and not difficult to prove by induction (see e.g. [18]), so in the following we assume $k \geq 2$. For a given integer $n$, let $r=R_{k}(n)$. Using the definition of the function $N_{k}(r)$ and Claim 2.5.3, we have

$$
n \geq N_{k}(r) \geq k \cdot\left(2^{r / k}-1\right),
$$

with both inequalities being sharp iff $n=N_{k}(r)$ and $r \bmod k=0$. From Claim 2.5.2, we can derive that the latter two conditions are in turn equivalent to the requirement that $n=k \cdot\left(2^{j}-1\right)$ for some $j \in \mathbb{N}$.

With $r=R_{k}(n)$, we can solve Ineq. (12) after $r$ to get $R_{k}(n) \leq k$. $\left(1+\log \frac{n}{k}\right)$, with equality iff $n=k \cdot\left(2^{j}-1\right)$ for some $j \in \mathbb{N} \backslash\{0\}$. This completes the proof of Theorem 2.5.

The above result settles the relation between balanced separator number and cycle rank. We turn our attention to the other end of the chain of inequalities in Theorem 2.1, namely to the relation between balanced separator number and treewidth. Here the optimal inequality will follow by refining a known result.

Recall that a graph is called chordal iff every cycle $C$ of order greater than 3 in $G$ has a chord, i.e., an edge connecting two vertices not adjacent in $C$. In other words, a chordal graph has no induced cycle of order greater than 3 .

In the following, we derive a strengthened version of a theorem originally due to Gilbert et al. [10] — these authors employed a looser notion of balanced separator.

Theorem 2.6. Let $G$ be a chordal graph whose largest clique has order $p$. Then $G$ contains a clique of order at most $p-1$ that is a balanced separator for $G$. 
Proof. The proof follows the one given by Gilbert et al. [10] for a similar statement. If $G$ itself is a clique, then removing a single vertex yields a balanced separator of order $|V(G)|-1=p-1$. So we assume in the following that $G$ is not a clique.

Choose $C$ to be the clique in $G$ which minimizes the maximum order among the connected components of $G-C$; in case there are several such cliques, take $C$ itself to be of minimum order among these cliques. Our aim is to show that then $C$ is a balanced separator for $G$. Let $A$ be a component of $G \backslash C$ that is of maximum order. The capstone of the original proof consists of deriving the following:

Fact. [10] Component $A$ contains a vertex $v$ adjacent to all vertices in $C$.

For a proof of this, the reader is referred to [10, Fact 3]. Notice that this fact implies that $|C| \leq p-1$, and thus it only remains to show that $|A| \leq\left\lceil\frac{|V(G)|-|C|}{2}\right\rceil$. For the sake of contradiction, assume this is not the case. Then, letting $B=V(G) \backslash(C \cup A)$, we must have $|A|>|B|$, and thus $|B| \leq|A|-1$. Take a vertex $v \in A$ that is adjacent to all vertices in $C$. Then $G-(C \cup\{v\})$ falls apart into the disconnected subgraphs $G[A]-v$ and $G[B]$, and the maximum order among the connected components of $G-(C \cup\{v\})$ is at most $|A|-1$. Thus, the clique $C \cup\{v\}$ would have been preferable to $C$, a contradiction.

It is well known that if a graph has treewidth at most $k$, then $G$ is subgraph of some chordal graph whose largest clique is of order at most $k+1$, compare $[1,22]$. The following corollary is now immediate:

Corollary 2.7. For any graph $G$, we have $\mathrm{s}(G) \leq \operatorname{tw}(G)$.

Again, for every possible value of $\mathrm{s}(G)$, there are infinitely many graphs for which this bound is tight, as witnessed by the $k$ th power of a path $P_{n}^{k}$. Also, the inequality is tight in the case of trees. In this way, Corollary 2.7 gives a proper generalization of Jordan's classical result on balanced separators in trees [14]. Since $\tilde{\mathrm{s}}(G) \leq \mathrm{s}(G)+1$, Corollary 2.7 also implies the inequality $\widetilde{\mathrm{s}}(G) \leq \operatorname{tw}(G)+1$ given by Robertson and Seymour [21, Proposition 2.5]. Observe that the latter inequality is off by 1 for all trees containing a path of order 4 .

Taking the statements of Lemma 2.3, of Theorem 2.5, and of Corollary 2.7 together, we have the following improvement over Theorem 2.1: 
Theorem 2.8. Let $G$ be a graph of order $n \geq 2$, and let $\mathrm{s}(G)$ denote its balanced separator number, let $\operatorname{tw}(G)$ and $\operatorname{pw}(G)$ denote its treewidth and pathwidth, respectively, and let $\mathrm{r}(G)$ denote its cycle rank. Then

$$
\mathrm{s}(G) \leq \mathrm{tw}(G) \leq \operatorname{pw}(G) \leq \mathrm{r}(G) \leq \mathrm{s}(G) \cdot\left(1+\log \frac{n}{\mathrm{~s}(G)}\right) .
$$

\section{Applications}

An immediate consequence of Theorem 2.8 is a tight upper bound for the cycle rank of a graph in terms of its treewidth:

Corollary 3.1. Let $G$ be a graph of order $n \geq 2$, and let $\operatorname{tw}(G)$ and $\mathrm{r}(G)$ denote its treewidth and its cycle rank, respectively. Then

$$
\mathrm{r}(G) \leq \operatorname{tw}(G) \cdot\left(1+\log \frac{n}{\operatorname{tw}(G)}\right) .
$$

Quite obviously, an analogous statement holds for cycle rank versus pathwidth. Our next application concerns the relation between the cycle rank and another width parameter for graphs, namely the bandwidth. The latter is defined in the following.

A linear layout of a graph $G$ of order $n$ is a bijection $\ell$ of $V(G)$ into the integer interval $[1 ; n]$. The bandwidth of a linear layout $\ell$ is defined as $\max _{\{u, v\} \in E(G)}|\ell(v)-\ell(u)|$; and the bandwidth of $G$, denoted by bw $(G)$, is defined as the minimum bandwidth among all linear layouts for $G$.

The bandwidth of a graph is known to be bounded below by its pathwidth, see e.g. [1, Thm. 44], so the latter is a common lower bound for both cycle rank and bandwidth. But how are these two related? It is not difficult to prove that the bandwidth of the star graph $K_{1, n}$ on $n+1$ vertices equals $\left\lfloor\frac{n+1}{2}\right\rfloor$, cf. [22]. On the other hand, it is clear that $\mathrm{r}\left(K_{1, n}\right)=2$, so there seems to be no interesting upper bound on the bandwidth of a graph in terms of its cycle rank. For the converse direction, we obtain the following result as a corollary to Theorem 2.4:

Corollary 3.2. Let $G$ be a graph of bandwidth at most $k$. Then

$$
\mathrm{r}(G) \leq R_{k}(n),
$$

and this bound is tight if $G$ is the kth power of the path graph of order $n$. 
Proof. A basic fact about the bandwidth bw $(G)$ of a graph $G$ of order $n$ is that bw $(G) \leq k$ iff $G$ is isomorphic to a subgraph of $P_{n}^{k}$ [6]. Now we have $\mathrm{r}\left(P_{n}^{k}\right)=R_{k}(n)$, and the cycle rank can never increase when taking subgraphs.

As a final application, we determine a sublinear upper bound on the cycle rank of the $d$-dimensional hypercube. Recall that the vertex set of the $d$-dimensional hypercube is $\{0,1\}^{d}$, and two vertices are adjacent, if their vector representations differ in exactly one coordinate. Bounding the cycle rank of this graph from below has turned out to be useful in formal language theory, namely for comparing the relative succinctness of different variants of the regular expression formalism, see [12].

Theorem 3.3. Let $H_{d}$ denote the d-dimensional hypercube of order $n=2^{d}$. Then

$$
\mathrm{r}\left(H_{d}\right)=O\left(\frac{n \log \log n}{\sqrt{\log n}}\right) .
$$

Proof. Harper [13] proved that for the bandwidth of the $d$-dimensional hypercube holds

$$
\mathrm{bw}\left(H_{d}\right)=\sum_{i=0}^{d}\left(\begin{array}{c}
d \\
\lfloor/ 2\rfloor
\end{array}\right) .
$$

Using Stirling's approximation, one can show that this sum is in $\Theta\left(d^{-1 / 2} 2^{d}\right)$. To estimate the cycle rank of this graph, we use Corollary 3.2 and Theorem 2.5 to obtain:

$$
\begin{aligned}
\mathrm{r}\left(H_{d}\right) & \leq \mathrm{bw}\left(H_{d}\right) \cdot \log \left(\frac{2^{d}}{\mathrm{bw}\left(H_{d}\right)}\right) \\
& =O\left(d^{-1 / 2} \cdot 2^{d} \cdot \log \frac{2^{d}}{\Theta\left(d^{-1 / 2} 2^{d}\right)}\right) \\
& =O\left(\frac{n \log \log n}{\sqrt{\log n}}\right),
\end{aligned}
$$

as desired.

As observed in [12], the hypercube of order $n$ has cycle rank at least $\Omega\left(\frac{n}{\sqrt{\log n}}\right)$, so this upper bound is tight up to a factor of $O(\log \log n)$. In contrast, utilizing the known fact that the pathwidth of the hypercube is equal to its bandwidth [4] together with the previously known upper bound from Theorem 2.1 would result in an upper bound that even exceeds the trivial upper bound of $n$. 
Acknowledgement The author would like to thank Petra Scheffler for sending him a copy of [22] and [23].

\section{References}

[1] H. L. Bodlaender. A partial $k$-arboretum of graphs with bounded treewidth. Theoretical Computer Science, 209:1-45, 1998. MR1647486

[2] H. L. Bodlaender, J. S. Deogun, K. Jansen, T. Kloks, D. Kratsch, H. Müller, and Zs. Tuza. Rankings of graphs. SIAM Journal on Discrete Mathematics, 11(1):168-181, 1998. MR1612885

[3] H. L. Bodlaender, J. R. Gilbert, H. Hafsteinsson, and T. Kloks. Approximating treewidth, pathwidth, frontsize, and shortest elimination tree. Journal of Algorithms, 18(2):238-255, 1995. MR1317666

[4] L. S. Chandran and T. Kavitha. The treewidth and pathwidth of hypercubes. Discrete Mathematics, 306(3):359-365, 2006. MR2204113

[5] C.-W. Chang, D. Kuo and H.-C. Lin. Ranking numbers of graphs. Information Processing Letters, 110(16):711-716, 2010. MR2676809

[6] V. Chvátal. A remark on a problem of Harary. Czechoslovak Mathematical Journal, 20(1):109-111, 1970. MR0266791

[7] J. S. Deogun, T. Kloks, D. Kratsch, and H. Müller. On the vertex ranking problem for trapezoid, circular-arc and other graphs. Discrete Applied Mathematics, 98(1-2):39-63, 1999. MR1723686

[8] R. Diestel. Graph Theory, volume 173 of Graduate Texts in Mathematics. Springer, 3rd edition, 2006.

[9] L. C. Eggan. Transition graphs and the star height of regular events. Michigan Mathematical Journal, 10(4):385-397, 1963. MR0157840

[10] J. R. Gilbert, D. J. Rose, and A. Edenbrandt. A separator theorem for chordal graphs. SIAM Journal on Algebraic and Discrete Methods, 5(3):306-313, 1984. MR0752037

[11] R. L. Graham, D. E. Knuth, and O. Patashnik. Concrete mathematics: A foundation for computer science. Addison-Wesley, 1989. MR1397498

[12] H. Gruber and M. Holzer. Tight bounds on the descriptional complexity of regular expressions. In V. Diekert and D. Nowotka, editors, 13th International Conference on Developments in Language Theory, volume 5583 of LNCS, pages 276-287. Springer, 2009. MR2544708

[13] L. H. Harper. Optimal numberings and isoperimetric problems on graphs. Journal of Combinatorial Theory, 1:385-393, 1966. MR0200192

[14] C. Jordan. Sur les assemblages de lignes. Journal für die reine und angewandte Mathematik, $\operatorname{LXX}(2): 185-190,1869$.

[15] M. Katchalski, W. McCuaig, and S. M. Seager. Ordered colourings. Discrete Mathematics, 142(1-3):141-154, 1995. MR1341442 
[16] T. Kloks. Treewidth, Computations and Approximations, volume 842 of Lecture Notes in Computer Science. Springer, 1994. MR1312164

[17] F. Manne. Reducing the height of an elimination tree through local reorderings. Technical Report CS-91-51, University of Bergen, Norway, 1991.

[18] R. McNaughton. The loop complexity of regular events. Information Sciences, 1(3):305-328, 1969. MR0249219

[19] J. Nešetřil and P. Ossona de Mendez. Tree-depth, subgraph coloring and homomorphism bounds. European Journal of Combinatorics, 27(6):1022-1041, 2006. MR2226435

[20] S. Novotny, J. Ortiz, and D. A. Narayan. Minimal $k$-rankings and the rank number of $P_{n}^{2}$. Information Processing Letters, 109(3):193-198, 2009. MR2485087

[21] N. Robertson and P. D. Seymour. Graph minors. II. Algorithmic aspects of tree-width. Journal of Algorithms, 7(3):309-322, 1986. MR0855559

[22] P. Scheffler. Die Baumweite als ein Maß für die Kompliziertheit algorithmischer Probleme. PhD thesis, Karl-Weierstraß-Institut für Mathematik, Dissertation A, Report RMATH-04/89. Akademie der Wissenschaften der DDR, Berlin, 1989. MR1004243

[23] P. Scheffler. Optimal embedding of a tree in linear time into an interval graph. In J. Nešetřil and M. Fiedler, editors, 4th Czechoslovakian Symposium on Combinatorics, Graphs and Complexity, pages 287-291. Elsevier, 1992. MR1206281

Hermann Gruber

KNOWLEDGEPARK AG

LEONRODSTR. 68

D-80636 MÜNCHEN

GeRmany

E-mail address: hermann.gruber@knowledgepark-ag.de

RECEIVED JANUARY 16, 2011 